\title{
Rumiación y abuso sexual infantil. Dolor cíclico y estrategias terapéuticas
}

\author{
Felipe Paredes Ramos ${ }^{1}$ y Alexis Bustos Villarroel ${ }^{2}$
}

Fecha de recepción: 12-08-2019

Fecha de aceptación:15-10-2019

\section{RESUMEN}

El presente artículo hace referencia a un elemento central del trabajo con adolescentes que han sobrevivido abuso sexual infantil (ASI) y se encuentran enfrentando reexperimentación traumática. Se realiza una revisión narrativa de las principales teorías respecto del fenómeno de la rumiación y sus efectos devastadores en la experiencia del dolor asociado a ASI. Se discute acerca del rol de este fenómeno en el desarrollo y la mantención de sintomatología asociada a estrés postraumático. Se delinean los principales efectos de la manifestación de este proceso en las personas y la relación con ASI. Finalmente, se aborda los aportes desde la terapia narrativa, ejemplificando, a través del trabajo con una adolescente, metodologías terapéuticas para acompañar a los adolescentes a salir del ciclo del dolor en el cual se ven sumergidos tras enfrentar procesos de rumiación asociados a ASI.

Palabras clave: abuso sexual infantil, reexperimentación traumática, rumiación, terapia narrativa

1 Psicólogo, MSc in Child and adolescent Mental Health at King's College London. Diplomado Internacional en Terapia Narrativa, para el trabajo con individuos, grupos y colectivos. Pranas, Prácticas Narrativas Santiago de Chile, en colaboración con Colectivo Prácticas Narrativas, México y Narrative Practices Adelaide, Australia. Excoordinador del Programa de Reparación de Maltrato Grave y Abuso Sexual Infantil, PRM CAVAS Regional de Valparaíso, convenio SENAME-PDI. Mail: felipeparedesramos@gmail.com

2 Asistente Social, Licenciado en Trabajo Social, Postítulo en Psicología Familia y Derecho, Postítulo en Psicología Jurídica al Servicio de la Investigación Forense y Criminal, Formación en Terapia Familiar Sistémica, Formación en Terapia Narrativa, Magíster en Filosofía mención Pensamiento Contemporáneo, Universidad de Valparaíso. Estudiante de Doctorado en Psicología Clínica y de la Salud, Universidad de Barcelona, España. Actualmente docente de la Escuela de Trabajo Social de la Universidad de Valparaíso. Docente de pre y posgrado en universidades de la zona centro del país y cofundador de Metáfora: Centro de Terapia del Trauma. Mail: bustosvillarroel@gmail.com - alexis.bustos@uv.cl 


\title{
Rumination and Child Sexual Abuse: The Cycle of Pain and Therapeutic Strategies
}

\author{
ABSTRACT
}

The present article focuses on a central element of the therapeutic work with adolescents that have survived child sexual abuse (CSA) and who are coping with traumatic flashbacks. The study implements a narrative review of the main theories concerning the phenomenon of rumination and its tremendous effect on the experience of pain associated with CSA. It discusses the role of rumination on the onset and maintenance of symptomatology associated with Post-Traumatic Stress Disorder and outlines the primary effects of rumination and the relationship between rumination and CSA. Finally, the principal inputs of narrative practices in the work on rumination are reviewed based on a case study of work done with an adolescent. Different conversational methodologies are applied to help the adolescent break the cycle of pain associated with the process of post-CSA rumination.

Keywords: child sexual abuse, traumatic flashbacks, rumination, narrative therapy

\section{Ruminação e abuso sexual infantil. Dor cíclica e estratégias terapêuticas}

\section{RESUMO}

\begin{abstract}
Este artigo faz referência a um elemento central do trabalho com adolescentes que sobreviveram ao abuso sexual infantil (ASI) e estão enfrentando reexperimentação traumática. Foi realizada uma revisão narrativa das principais teorias a respeito do fenômeno da ruminação e seus efeitos devastadores na experiência da dor associada ao ASI. É discutido o papel deste fenômeno no desenvolvimento e na manutenção da sintomatologia associada ao estresse pós-traumático. São apresentados os principais efeitos da manifestação deste processo nas pessoas e a relação com o ASI. Finalmente, são abordadas as contribuições da terapia narrativa, exemplificando, através do trabalho com uma adolescente, metodologias terapêuticas para auxiliar os adolescentes a saírem do ciclo da dor no qual se encontram submersos após enfrentar processos de ruminação associados ao ASI.
\end{abstract}

Palavras-chave: abuso sexual infantil, reexperimentação traumática, ruminação, terapia narrativa

\section{Introducción}

En la filosofía del budismo zen existe un término - makyo- para hacer referencia a las ilusiones que se crean durante el proceso 
meditativo. La imagen con la cual se explica este concepto, toma el remolino como un símbolo de la experiencia de una ilusión que genera dolor al tomar la conciencia y hacerla chocar con contenidos constantes que nos imbuyen y nos ocultan los otros ámbitos de la realidad, limitando la experiencia y encerrándonos en los contenidos de la ilusión, con una mezcla entre fascinación y horror.

La reexperimentación intrusiva posee similitudes con el concepto japonés de "ilusión", un adolescente que ha vivido una o múltiples experiencias de abuso o trauma será proclive a reexperimentar ideas intrusivas respecto de lo vivido, imbuyéndose en el dolor de forma atemporal, con escasos grados de conciencia de lo que le ocurre y será altamente posible que pierda la sensación de control respecto de su propia vida (Van der Kolk, 2016; Bustos y Paredes, 2018). La no predictibilidad de la ocurrencia de ideas intrusivas puede generar una experiencia cargada por la vulnerabilidad sentida, que favorecerá mecanismos de afrontamiento no adaptativos que terminan recrudeciendo el dolor y la sensación de amenaza (Ehlers y Clark, 2000).

La rumiación se constituye como un factor de riesgo para la aparición del Trastorno de Estrés Postraumático (Elwood et cols., 2009). De la misma forma, es uno de los factores de riesgo que tendría mayor asociación con la mantención y recrudecimiento de la sintomatología de estrés postraumático (Elwood et al., 2009).

En el presente artículo discutiremos los aportes teóricos que han intentado dar cuenta de dicha asociación. Usando un esquema similar al propuesto por Smith y Alloy (2009), describiremos los principales modelos sobre rumiación y sus aportes a la conceptualización del fenómeno, para luego presentar los principales efectos asociados a ésta. Posteriormente, nos centraremos en la rumiación y abuso sexual. Y, finalmente, describiremos aportes construidos desde la práctica y el acto de traducción a los procesos terapéuticos desde las prácticas narrativas (White, 2016; Epston y White, 1993). 


\section{El fenómeno de la rumiación}

La rumiación ha sido un fenómeno principalmente descrito en la literatura sobre depresión. Beck, padre de la psicología cognitiva, realiza una primera aproximación al concepto basándose en una definición que toma los contenidos reiterativos de los pensamientos de personas depresivas (Beck, 1967). No obstante, es Susan Nolen-Hoeksema, de la Universidad de Yale, la que inicia un largo proceso de estudio respecto de la rumiación, cambiando el foco desde una investigación sobre la depresión basada en contenidos reiterativos, a una investigación que subraya el proceso de pensamientos reiterativos (Nolen-Hoeksema, 1991). Define "rumiación" como el acto de pensar, repetitiva y pasivamente, acerca de las emociones negativas, precipitadoras de eventos negativos, síntomas de distrés y preocupación acerca del significado del distrés (Nolen-Hoeksema, 1991). Su definición es la más usada en la literatura sobre depresión y la explicaremos en detalle, ya que su trabajo es la piedra angular desde la que se ha reflexionado sobre el fenómeno de la rumiación y su teoría sigue vigente en investigaciones hasta hoy.

La teoría de Nolen-Hoeksema, denominada Responses Styles Theory (teoría de los estilos responsivos), se basa en la investigación sobre vulnerabilidades respecto de la depresión (Smith y Alloy, 2008). Durante estas investigaciones, su equipo observó patrones cognitivos característicos en los que personas que tendían a mantener episodios disfóricos y episodios de depresión mayor, presentaban estilos de pensamientos similares. La conexión de dichos pensamientos se daba en procesos equivalentes más que en contenidos. Estos procesos presentaban una sobre involucración o autoconciencia (self-awareness, en inglés) en aspectos negativos de la experiencia (Nolen-Hoeksema, 2010). Su definición, por ende, se centra en el proceso perseverativo de pensamientos acerca de los sentimientos y problemas (Nolen-Hoeksema, 2008), alejándose de la visión más clásica del pensamiento cognitivo conductual, sobre el contenido de esquemas de pensamientos automáticos y estilos cognitivos negativos (Beck, 1967). 
La teoría de los estilos responsivos plantea que, frente a estados anímicos depresivos, una persona puede enfrascarse en patrones de pensamientos reiterativos que tienden a tener tres posibilidades o puntos focales: autocentradas, centradas en la sintomatología o centradas en las posibles consecuencias y causas del estado anímico percibido (Nolen-Hoeksema, 1991). La rumiación centrada en sí mismo se caracteriza por una focalización en el sentido del estado anímico en la construcción de autoimagen. Preguntas tales como: “¿por qué reacciono de esta manera?”, “ipor qué soy así?” son características de la rumiación centrada en sí mismo, en la que las respuestas o conclusiones tienden a ser devastadoras y perpetúan estados anímicos depresivos, en vez de ayudar a salir de ellos. La rumiación centrada en la sintomatología, asociada a los estados anímicos depresivos, se centra en los efectos concretos de los estados anímicos en la vida de las personas; por ejemplo, "piensa en lo difícil que es concentrarse o lo difícil que es levantarse", acto que no invita a la acción concreta, sino que, a su vez, perpetúa el mantenerse en una condición. Finalmente, la rumiación centrada en las posibles consecuencias y causas del estado anímico, focaliza el pensamiento en preguntas asociadas a las razones por las cuales el estado anímico invade la experiencia del sujeto y/o en los efectos de la experimentación de alguna sintomatología dada. Por ejemplo: "no voy a poder realizar mi trabajo si no salgo de este estado anímico", o “ipor qué me pasan estas cosas a mí?". Ideas como esta permean la experiencia del sujeto con ansiedad, mayor tristeza y miedo (Nolen-Hoeksema, 2008).

Cada uno de estos procesos pudiese considerarse como pensamientos normales tras enfrentarse a una situación que cause distrés. No obstante, Nolen-Hoeksema plantea en su teoría un continuo, en el cual el extremo pernicioso de este proceso tendría como características centrales la rigidez del proceso y el efecto directo en el aumento del estado anímico depresivo, con un consecuente resultado en la inmovilidad del sujeto para cambiar su situación (Sarin y Nolen-Hoeksema, 2009). 
En relación con la rigidez, Nolen Hoeksema plantea que está asociada con inflexibilidad cognitiva y con dificultad para cambiar la atención desde estímulos negativos (Davis y Nolen-Hoeksema, 1999). La autora plantea que esta rigidez tendría como mediador fundamental la sensación que los sujetos poseen en este proceso, esta es que, al inmiscuirse en estos pensamientos rumiativos, ganarían mayor capacidad de introspección respecto del significado de sus sentimientos y problemas, tratando de generar conexiones entre sus problemas, intentando discernir las razones por las cuales las situaciones le suceden, tratando de crear sentido de las memorias, las cuales son difíciles de integrar (Lyubomirsky y Nolen-Hoeksema, 1993), lo que haría de la rumiación un mecanismo que emerge como una respuesta, para intentar cobrar sentido de algo pernicioso ocurrido y difícil de integrar. Como plantea Mclaughlin, la rumiación parece estar asociada a un proceso sostenido de abordaje del material emocional negativo (Mclaughlin et al., 2007). No obstante, si bien es un proceso que, al parecer, está orientado a intentar dar respuesta, no posee la flexibilidad suficiente como para llegar a una solución que efectivamente regule la respuesta emocional, sino que, por el contrario, la aumenta. Investigadores sugieren que esto podría deberse a que el tipo de pensamiento presente en la rumiación tiende a estar asociado con un incremento en la actividad de la amígdala ${ }^{3}$ (Ray et al., 2005; Siegle et al., 2002). Ciertas investigaciones intentan diferenciar entre pensamiento autocentrado, focalizado en aspectos negativos, y el pensamiento autocentrado reflexivo (Joormann et cols., 2006), lo cual genera una interesante distinción respecto de la efectividad de esta estrategia de enfrentamiento, en la cual la rigidez vuelve a ser un elemento central.

Otro elemento que ha sido asociado a la tendencia al pensamiento autocentrado con efectos perniciosos es un pensamiento hiper-

3 La amígdala cerebral es una estructura subcortical, situada en la parte interna del lóbulo temporal medio. Posee conexiones con la mayoría del encéfalo, pudiendo con ello afectar al conjunto del sistema nervioso. Se la relaciona con la regulación de las emociones. 
crítico. Watkins (2004), en su estudio, plantea que individuos que se focalizan pasivamente y de forma crítica en su estado de ánimo, tienen peores resultados que aquellos a quienes se les invita a reflexionar sobre su ánimo. Otro estudio (Kross, Ayduk y Mischel, 2005) indica que aquellos individuos que se focalizaron en sí mismos, pero evaluaron las razones para sus sentimientos, lograron mejores resultados que aquellos que lo hicieron de forma pasiva y crítica. Burwell et al. (2007) plantean que el concepto de "rumiación" debe ser descompuesto en dos conceptos o formas de pensamiento autocentrado; Brooding, que se referiría al pensamiento autocentrado con características pasivas; autocrítico, que se asociaría en la adolescencia con estrategias de enfrentamiento de evitación, negación y fuga. Por otro lado, el pensamiento autocentrado reflexivo que, según su estudio, se relacionaría con mecanismos de enfrentamiento de tipo I, asociados con soluciones efectivas para las dificultades.

Otro modelo que ha influenciado la conceptualización con relación a la rumiación es el modelo basado en la tristeza (Conway, Csank, Holm y Blake, 2000). Este modelo define "rumiación" como pensamientos repetitivos acerca de la tristeza. El modelo nace de la crítica al modelo responsivo, en cuanto las medidas que utiliza tienden a estar interrelacionadas y, por ende, según los autores, no se centraría en la rumiación. Según Conway et al. (2000) el Response Styles Questionnaire (Nolen-Hoeksema y Morrow, 1991) solo hace referencia específica a un apartado o factor a rumiación. Por este motivo, construyen una prueba llamada Rumination on Sadness Scale (RSS). Uno de los elementos interesantes de este modelo reside en que pone énfasis en el elemento tiempo. Al definir rumiación desde una comprensión que emerge desde el estudio de la vulnerabilidad de la depresión, definen también los pensamientos repetitivos desde un contexto que alude a circunscribir dicha repetición sobre el distrés que sucede en eventos en el presente. Investigaciones al respecto tienden a desacreditar dicha noción, mostrando una flexibilidad en torno a los contenidos que pudiesen ser propios de la rumiación. Investigaciones asociadas a 
los contenidos de los procesos rumiativos (McLaughlin, Borkovec y Sibrava, 2007) tienden a mostrar que este fenómeno pudiese estar circunscrito a elementos en el pasado y presente, y que la orientación temporal de los pensamientos repetitivos pudiese ir variando a lo largo del curso de la rumiación, en la que un individuo pudiese iniciar con pensamientos respecto del pasado, pero incrementar el foco en pensamientos relacionados con el presente y el futuro durante el curso de un proceso rumiativo.

Un tercer modelo emergente, a partir de la investigación centrada en la vulnerabilidad a la depresión, es el modelo Stress Reactive Rumination (Alloy et al., 2000). Focaliza su conceptualización sobre rumiación relacionándola con experiencias asociadas con una experiencia traumática. Este modelo presenta la ventaja respecto de los otros modelos, en cuanto captura la presencia del fenómeno de rumiación como un fenómeno que puede darse antes de la presencia de afecto negativo. Es decir, este modelo propone, como elemento enriquecedor, la rumiación como causante de afecto negativo (Smith y Alloy, 2009). Lo interesante de esta propuesta es que complejiza la relación entre afecto negativo y rumiación, mostrando su bidireccionalidad en torno a la asociación entre ambos fenómenos. No obstante, una potencial limitación de este modelo es que propone que la rumiación consiste solamente en pensamientos asociados a los eventos traumáticos o estresores. Este punto es importante ya que, en la experiencia clínica, los pensamientos rumiantes tienden a poseer una flexibilidad en contenidos, que provee una vulnerabilidad para ideas intrusivas relacionadas con trauma o eventos estresantes, $y$, por ende, no necesariamente deben centrarse en potenciales contenidos asociados a trauma o estrés (Bustos y Paredes, 2018).

Un cuarto modelo - Goal Progress Theory o teoría de progresión de objetivos (Martin, Tesser y McIntosh, 1993)_, proveniente de la investigación asociada a autorregulación, aporta una interesante propuesta respecto de la rumiación no asociada a un estado anímico, sino como una respuesta al fracaso de progresar satisfactoriamente hacia un objetivo. Este modelo propone una reflexión 
en torno a las metacogniciones asociadas a la rumiación. A través de su test SMRI (Scott McIntosh Rumination Inventory), hacen énfasis en la motivación asociada a la rumiación, tomándola como una medida que estaría vinculada. Si bien esta teoría se centra en la rumiación como un malestar en torno a expectativas, el hecho de que se focalicen en las motivaciones y metacogniciones provee como un elemento central a evaluar respecto de la experiencia de malestar asociada a la rumiación. Este será un punto central en el presente artículo y posteriormente ahondaremos en esta hipótesis, en cuanto a una de las practicas narrativas llamada lo ausente pero implícito (White, 2016).

Un quinto modelo, denominado Self-Regulatory Executive Function (Wells y Matthews, 1994, 1995), define la rumiación como un proceso asociado a la respuesta frente a la discrepancia entre un estado actual y un estado deseado. Inserto dentro de un modelo mayor, en relación con los denominados "desordenes emocionales”, los cuales incluyen atención, regulación cognitiva y creencias sobre estrategias de regulación emocional, el modelo integra creencias metacognitivas en la conceptualización de la rumiación, la cual juega un rol más amplio en su desarrollo como un estilo responsivo (Smith y Alloy, 2009). La teoría presenta un aporte en relación con el modelo de Goal Progress Theory, en cuanto incluye no solo la motivación y las metacogniciones asociadas al uso de la rumiación, sino que, a la vez, propone las creencias respecto de los efectos de la rumiación como un elemento importante de su uso como mecanismo de regulación. Este aporte ayudaría a la diferenciación de una rumiación centrada en lo positivo vs una centrada en lo negativo.

\section{Efectos de la rumiación}

Hasta este apartado hemos descrito modelos teóricos y principales autores asociados a la rumiación, como también los distintos aportes de cada teoría al desarrollo de este concepto, en los que puede evidenciarse la complejidad de las comprensiones teóricas con respecto al mismo y la cantidad de formas de intentar medir 
dicho fenómeno. En el presente apartado haremos una revisión respecto de los principales efectos estudiados de la rumiación y las consecuencias que los estudios en la temática, independiente del marco teórico, han logrado asociar.

En un primer lugar, la rumiación tendría un efecto directo en el aumento y exacerbación de los pensamientos centrados en aspectos negativos de la autoimagen (Nolen-Hoeksema, Blair y Lyubomirsky, 2008). Uno de los principales efectos de la rumiación es que pareciera tener un efecto amplificador de los estados disfóricos, generando mayor pensamiento negativo, ya sea respecto del pasado, el futuro o el presente. White (2016) plantea que este proceso ocurre tras un esfuerzo por resolver los problemas, pero centrado en ideas acerca de la propia imagen basada en aspectos negativos de la autoimagen. Esto se condice con la investigación en rumiación por cuanto, en experimentos, personas con evaluación de estados disfóricos, que han sido inducidas a rumiar de forma espontánea, tienden a recordar memorias negativas de su pasado de mayor forma que aquellos que han sido inducidos a distraerse de pensamientos negativos (Lyubomirsky et al., 1998). De la misma forma, aquellos individuos que presentan rumiación disfórica, inducidos a rumiar, tienden a hablar acerca de aquellos problemas que los aquejan tal como conflictos con la familia. En cambio, aquellos pacientes no disfóricos y aquellos que presentan rumiación disfórica, pero que fueron inducidos a distraerse de pensamientos negativos tendieron a hablar más sobre situaciones con contenidos más positivos (Lyubomirsky et al., 1999). El equipo de Nolen-Hoeksema (1995) propone que las personas que tienden a centrarse en la rumiación como mecanismo de afrontamiento de emociones negativas, tienden a caracterizarse por priorizar pensamientos más negativos, por tener más pensamientos autocríticos, a culparse a sí mismos por sus problemas presentes y a expresar un reducido optimismo y autoconfianza para lograr resolver estos problemas. Un interesante experimento de este equipo presentó eventos negativos hipotéticos a personas tendientes a rumiar, evidencian- 
do una tendencia a preenjuiciar dichos eventos, minimizando el éxito y sobre generalizando los fracasos.

Un segundo e importante efecto de la rumiación sería que afecta a la capacidad de resolver problemas efectivamente (NolenHoeksema, Blair y Lyubomirsky, 2008). Investigaciones al respecto muestran que las personas que tienden a rumiar, como hemos dicho, dicen estar tratando de resolver y entender sus problemas (Papageorgiou y Wells, 2001). Esta creencia acerca de esta particular estrategia, unida a la motivación por solucionar algo que genera molestia, parece impedir e interferir con la resolución de problemas. Experimentos de inducción de rumiación tienden a evidenciar que las personas con tendencia a rumiar evalúan los problemas como abrumadores y sin resolución, dificultando el proceso de crear soluciones efectivas (Lyubomirsky et al., 1999). White, describiendo la externalización de ideas y el efecto de las ideas rígidas, negativas y repetitivas con respecto a la propia identidad, lo plantea de la siguiente manera: "Hay algo irónico allí. Muy a menudo, estos mismos entendimientos internos (y acciones que conforman), están, desde un inicio, implicados de manera primordial en el desarrollo de los problemas" (White, 2016, p. 48). Investigaciones acerca de los efectos en pacientes diagnosticados con depresión muestran resultados similares (Donaldson y Lam, 2004; Watkins y Baracaia, 2002; Watkins y Moulds, 2005). Incluso, cuando una persona con tendencia a usar la rumiación genera un plan de solucionar efectivamente un problema, la rumiación tiende a impedir que logre implementarla (Lyubomirsky et al., 1999). Esto puede deberse a que las personas tendientes a rumiar confían menos en que la solución que han generado a un problema complejo tenga efecto.

En tercer lugar, uno de los efectos directos de la rumiación se relaciona con la inhibición del comportamiento instrumental, concepto referido a la actual toma de acciones asociadas a la resolución de problemas, realizando una diferencia clara con respecto al efecto anterior, en cuanto no implica la planificación y creación de soluciones (Nolen-Hoeksema, Blair y Lyubomirsky, 2008). 
Los autores plantean que la rumiación puede interferir en la solución de problemas y las acciones asociadas, a través de un ataque directo a la motivación de las personas y a la iniciativa. La rumiación, a través de la promesa de una solución, mantendría el foco en los estados anímicos depresivos, lo cual tendría como efecto el convencimiento de una falta de eficacia y una invitación a no participar en comportamientos que pudiesen ser constructivos para la vida de las personas (Nolen-Hoeksema, Blair y Lyubomirsky, 2008). Investigaciones muestran que, aunque las personas que rumian tienden a reconocer actividades que son placenteras y que potencialmente ayudarían a elevar el ánimo, no iniciarían acciones tendientes a seguirlas (Lyubomirsky y Nolen-Hoeksema, 1993; Wenzlaff, Wegner y Roper, 1988). Modelos terapéuticos, tales como Behavioral Activation (Veale, 2008), hacen eco de estos descubrimientos y están mostrando efectividad en la intervención asociada a depresión, al tener como una de sus principales actividades el acompañar a las personas a realizar este tipo de acciones de forma supervisada. No obstante, se cree que modelos como Behavioral Activation y terapia cognitiva conductual, no logran acompañar el proceso de agencia personal debido al componente educacional que se encuentra en sus bases teóricas, lo que genera una intervención destinada a enseñar a la persona y no a que esta logre empoderarse de sus acciones (Bustos y Paredes, 2018). Sin embargo, la inhibición instrumental es un elemento central a tener en cuenta, ya que evidencia procesos motivacionales que pueden estar afectando a las personas y las consecuencias pueden ser sumamente peligrosas en cuanto a que, si bien puede existir una gradualidad en relación a la inacción, en el lado más pernicioso pudiese implicar estados catatónicos o fundamentar ideación suicida o autoinjurias.

Finalmente, uno de los efectos que genera mayor disrupción en la vida de las personas es que la rumiación genera una reducción paulatina del apoyo social (Nolen-Hoeksema, Blair y Lyubomirsky, 2008). Personas que tienden a rumiar se relacionan de una forma que es contraproducente con las personas que las rodean. No- 
len-Hoeksema y Davis (1999), en un estudio en adultos, encontraron que personas tendientes a la rumiación eran más proclives a buscar ayuda social de otros. No obstante, reportaban mayores problemáticas con dichas personas y una sensación de menor apoyo social. Estudios posteriores indicaron que esto podría deberse a la repetición continua de argumentos negativos sobre acontecimientos, lo que no les permite visibilizar las relaciones sociales en las que se encuentran inserto y, también, el posible apoyo recibido de otras personas tiende a generar cansancio y frustración frente a la continua necesidad de conversar sobre los mismos temas de pérdida y afectos negativos (Nolen-Hoeksema y Larson, 1999). En la experiencia clínica, tal pérdida de apoyo genera vulnerabilidades para procesos de polivictimización, y en consecuencia, en población adolescente que utiliza la rumiación como estrategia para lidiar con afectos negativos, podría significar aislamiento y, a su vez, menores redes sociales para enfrentar situaciones de hostigamiento de pares u otros grupos. Otra de las formas de enfrentar los procesos rumiativos, y que ayuda a la generación de este contexto de soledad, es la tendencia para lidiar con la rumiación o intentar parar este proceso mediante la agresión, ya sea hacia sí mismo o hacia otros (Zhan et al., 2017). Esto tendría como efecto el alejamiento de las personas, aumentando el bajo apoyo social.

\section{Rumiación y abuso sexual}

Las personas que han experimentado traumas severos presentan mayor tendencia a rumiar (Sarin y Nolen Hoeksema, 2010). De acuerdo con la teoría de los estilos responsivos, el desencadenante más común para rumiar es la experiencia de distrés o afecto negativo (Nolen-Hoeksema, 1991). Spasojevic y Alloy (2002) proponen que la tendencia a rumiar puede desarrollarse con mayor probabilidad en algunos grupos que en otros, tales como aquellos que han experimentado eventos traumáticos en su vida y que son difíciles de explicar o comprender. Entre los posibles traumas complejos de explicar, el abuso sexual infantil presenta características propias que lo hacen más proclive a asociarse a 
la rumiación. La investigación demuestra que los niños y niñas que han sido víctimas de abuso sexual tienden a presentar altos niveles de distrés, impotencia y desesperanza, dada la dificultad para comprender las causas de tal vulneración, especialmente en casos en los que el agresor es conocido para la persona (Cicchetti y Toth, 2005; Spasojevic y Alloy, 2002). Este proceso genera vulnerabilidad, y las personas tienden a rumiar de mayor forma, focalizándose en aspectos negativos de la identidad y sobre todo en el distrés, como un intento desesperado para entender y controlar los resultados negativos de las emociones. No obstante, el resultado es sumergirse en un ciclo de dolor continuo, en el que - se cree- se genera un proceso que permea y posibilita la emergencia de memorias intrusivas (Bustos y Paredes, 2018). Hipotetizamos que, este proceso, se genera, a partir de la búsqueda activa de soluciones como motivador principal, una rebeldía hacia lo acontecido, que invita a hacer un acto de pararse frente a lo sucedido, como un gesto que denota la importancia de la experiencia, que denota lo relevante de no dejar pasar, lo relevante de lo sufrido, pero que, al no encontrar una respuesta coherente a lo sucedido o encontrar una que no solo se centre en los aspectos negativos y dolorosos de la autoimagen, provoca mayor dolor. El fenómeno de las ideas intrusivas voluntarias (Ehlers, Hackmann y Tanja, 2004) posee una conexión con dicho proceso, al traer a la conciencia imágenes de lo acontecido, como una forma de remirar imágenes traumáticas en búsqueda de las preciadas explicaciones. No obstante, pareciese que este proceso conectaría la experiencia de un evento pasado a la experiencia de un evento presente, generando una reexperimentación traumática no voluntaria. A dicho proceso nos referimos como el ciclo del dolor y, tal como se planteaba en la introducción, preferimos imaginarnos dicho proceso como un huracán que tiene como principal característica el viento girando sobre sí mismo. Aquella metáfora intenta rescatar la condición temporal de la rumiación, pero también la fuerza y los efectos devastadores de la misma. 
Otro elemento asociado a la relación entre abuso sexual y rumiación es la regulación emocional, aquel proceso por el cual un individuo logra mediar los efectos de emociones una vez reconocidas, nombradas y expresadas (Aldao et al., 2010). Para lidiar con emociones negativas, los sobrevivientes de abuso sexual infantil son proclives a utilizar la rumiación con el fin de manejar los efectos de emociones negativas (Sarin y Nolen-Hoeksema, 2010). Esta tendencia se relacionaría con las dificultades en la regulación emocional en quienes han vivenciado trauma recurrentemente, especialmente en la modulación de emociones críticas (Sarin y Nolen-Hoeksema, 2010). Concatenándolo con el proceso descrito, el efecto constante de reconectarse con el dolor cíclico afectaría la capacidad de regularlo y, por ende, aumentaría la posibilidad de desarrollar cuadros clínicos tales como depresión, estrés postraumático o cuadros ansiosos (Coyle et al., 2014). Hipotetizamos que dicha dificultad en la regulación sería un proceso bidireccional con el uso de la rumiación, en un intento desesperado por evadir el dolor asociado a las memorias intrusivas y a las significaciones asociadas, tanto con el fenómeno de sufrir con ellas y con las propias conclusiones respecto de la experiencia. Por ende, no solo sería un factor de inicio (onset) de cuadros clínicos, sino que también emergería como un factor de mantención de dichos cuadros (Elwood et cols., 2009). Investigaciones muestran que existen emociones mayormente vinculadas con las experiencias de abuso sexual en la infancia. Coyle et al. (2014), en su estudio en adultos que sufrieron ASI, plantean que existe una asociación entre la intensidad de las experiencias de tristeza, miedo y asco. Siendo esta última emoción una que no se ha estudiado con anterioridad en relación con su vinculación y regulación de experiencias de ASI. Coyle et al. (2014) proponen que existiría, inclusive, una asociación entre tristeza y mecanismos de afrontamiento de tipo evitativo, y el miedo y el asco con mecanismos de afrontamiento de hiperreactividad. No obstante, los procesos cognitivos y emocionales asociados al aumento en la intensidad de la percepción sobre dichas emociones aún no se han estudiado. 


\section{Rumiación en ASI como un factor de riesgo}

Múltiples estudios han dado cuenta de la asociación entre el uso de la rumiación en ASI y problemas asociados a conductas que pudiesen considerarse de riesgo (Sarin y Nolen-Hoeksema, 2010). Ya mencionamos la rumiación en ASI en relación con cuadros clínicos en los que principalmente ha sido asociada con cuadros ansiosos, estrés postraumático y depresión. No obstante, ciertas investigaciones muestran aspectos conductuales asociados, que podrían ayudar a incrementar la comprensión de conductas que suelen ser problemáticas en la terapia con las personas que han experimentado ASI.

La rumiación en ASI ha sido asociada a factores de vulnerabilidad para conductas autolesivas, ideación e intentos suicidas (Sarin y Nolen-Hoeksema, 2010). Una de las teorías que el modelo responsivo propone se refiere a la sobre focalización sobre sí mismo, en aspectos negativos, en relación con un afecto depresivo. Esto amplifica el pensamiento negativo y genera un contexto de vulnerabilidad, para el uso de comportamientos evitativos de pensamientos negativos direccionados hacia sí mismo (Heatherton y Baumeister, 1991; Nolen-Hoeksema et al., 2008). En este sentido, se propone una función a los comportamientos autolesivos, como un acto evitativo del foco negativo en la propia autoimagen. En esta misma línea de pensamiento, Baumeister (1991) ha planteado que aquellos individuos que poseen tendencia a una conciencia de sí mismos de forma aversiva, estarán especialmente inclinados a comportamientos evitativos, tales como el uso de drogas, atracones de comida, autoinjurias, entre otras formas, como un comportamiento para escapar del estado desagradable. El mecanismo que influye en la efectividad de dichos comportamientos es que tal conducta entrega un escape potente de la autoconciencia, proveyendo una reducción temporaria de la autoconciencia mediante la facilitación de un estado cognitivo de baja autoconciencia (Baumeister, 1991; Heatherton y Baumeister, 1991). Este proceso se relaciona con lo que plantea Van der Kolk (2016) respecto de la dificultad y baja efectividad de algunas terapias centradas en 
un foco tipo Top Down, que hacen énfasis en el lenguaje como principal medio terapéutico, y en las que, implícitamente, existe la noción de insight, desde la tradición psicoanalítica, o la noción de aprendizaje de la terapia cognitiva conductual. La conducta evitativa poseería un componente experiencial de "liberación" de un estado, que poseería un potencial anclado en la vivencia directa de un conocimiento que vuelve más "verdadero" al sujeto que una recomendación hipotética con miras en el futuro, como lo sería un enfoque con componentes netamente psicoeducativos.

Otra asociación que se ha realizado en relación con la rumiación en ASI, es la conexión con el enojo (Zhan et al., 2017). Tal emoción, tiene el potencial de permitir, de otra forma, iniciar estrategias de evasión de pensamientos negativos centrados en la propia persona (Ibíd.). Este podría ser uno de los potenciales mecanismos de la relación entre ASI y trastornos conductuales, en los que la asociación ha sido fundamentada por estudios sistemáticos (Maniglio, 2015). No obstante, aún es un área que requiere mayor investigación, en orden a dar cuenta de procesos específicos de esta asociación.

\section{Terapia narrativa y rumiación}

Las prácticas narrativas, como posteriormente la denominan sus autores ${ }^{4}$, Michael White y David Epston (1993), se refieren a las prácticas sustentadas en una ética que White denomina "colaborativa" (White, 2002). Esta ética introduce una comprensión basada en el sociocontruccionismo (Gergen, 2007) y en el postestructuralismo (Foucault, 1973), desde la cual se entiende al ser humano en constante construcción y que es éste quien da sentido al mundo y, por ende, a la terapia. White y Epston siguen a Bruner en sus postulados sobre los actos interpretativos, comprendiendo que "Todo conocimiento requiere un acto de interpretación" (Bruner, 1986, citado en Epston y White, 1993). Desde este postu-

4 La terapia narrativa inicialmente es conocida como un enfoque, luego se posiciona como terapia y, posteriormente, como práctica narrativa (White, 2007). 
lado, fundamentan el uso de la analogía del texto en la terapia para comprender a las personas.

Desde las prácticas narrativas, entonces, la persona es considerada como una posibilidad de cambio constante, en tanto no estaría definida ni por sus condicionantes biológicos, sociales o culturales, sino íntimamente relacionada con su historia y con las historias que cuentan de él o de ella. Por "historia" se comprendería el relato de una serie de eventos vinculados en una secuencia, a través del tiempo y en torno a un tema y una trama (Latorre, en García, 2013).

La terapia narrativa intentaría desentrañar las historias que saturan el problema que aqueja a la persona, con el fin de generar una separación entre las personas y el problema (Payne, 2000). Tal separación, llamada "externalización", es también otra ética fundamental en narrativa, la cual permitiría generar un territorio desde donde considerar el problema como una entidad separada de la persona. Para White (2016), tal acto se basaría en la comprensión proveniente del postestructuralismo, en el que los discursos dominantes, desde donde se sustenta el dolor de la persona, provienen de prácticas de fragmentación que, mediante la adscripción o compartimentalización de los relatos sobre sí misma, concluyen en verdades que abarcan completamente la identidad de la persona, asignándoles identidades deterioradas. El objetivo de la externalización sería, por ende, ayudar a separar la identidad de la persona de la identidad del problema (White, 2016). No obstante, dicha separación no alude a que la persona se deslinde de su responsabilidad respecto de las dificultades en las que se encuentra, sino más bien la invita a responsabilizarse, pero desde un sitial desde el que pueda visualizar dicha relación. La analogía de territorio seguro, aludiendo a una analogía territorial $\left(\operatorname{mapas}^{5}\right)$, es una me-

5 La noción de "mapa" debe ser entendida en dos aspectos a lo menos. El primero de ellos es la analogía propuesta por la antropóloga Barbara Myerhoff: "el mapa no es el territorio", y, segundo, "mapa" entendido como una posibilidad de abordaje y de abrir conversaciones. No debe ser entendido como un mapa cualquiera de narrativa, por ejemplo, el mapa de reautoría, como la técnica de reautoría. Además, 
táfora que facilita la comprensión del modelo, en cuanto la construcción de una historia alternativa a las historias saturadas del problema permite identificar los conflictos, reconocer y evaluar los efectos y tomar posición respecto del problema (White, 2016).

La externalización se configura como una práctica adecuada para el abordaje de la rumiación, ya que, mediante preguntas que presentan un andamiaje, facilita que la persona paulatinamente tome distancia de los procesos en los cuales se ve sobre involucrado. El proceso de andamiaje del mapa de externalización, es un proceso que involucra nombrar los pensamientos rumiantes de una forma que tenga sentido para la persona. El proceso de nombrar los problemas implica iniciar conversaciones sobre el problema, de una forma tal que éste pueda mostrar cómo se desenvuelve en la vida de la persona. Conversaciones en el panorama de acción permiten concretizar las vivencias en relación con el problema, y es desde estas historias que se pide a la persona, una vez nombrado el problema, iniciar una descripción de los efectos de éste en su vida. La conversación de forma externalizada permite evitar el efecto de la rumiación como algo que invita a la culpa, tristeza, vergüenza o congelamiento. Es desde una descripción enriquecida de los efectos del problema que se invita a tomar una posición de evaluación del problema y sus efectos en la vida. También, a tomar una posición respecto de los sueños, deseos para su vida, motivaciones, esperanzas o, como White (2016) lo denomina, desde el "panorama de identidad". Por ejemplo, Catalina ${ }^{6}$ fue agredida sexualmente por su hermano, el cual a su vez abusó a tres hermanos menores que ella. Tras años de estar alejada físicamente de él, cuando inició el proceso terapéutico, era invadida por imágenes intrusivas en

el mapa contempla y entremezcla aspectos ontológicos, epistemológicos, teóricos, metodológicos, procedimentales, teleológicos y éticos. White y Epston han sido enfáticos en trazar la distinción fundamental entre mapa y técnica, en la cual esta última, en sí misma, emerge como una acción sin mayor mediación reflexiva o interpretativa; el mapa, por su parte, es una posibilidad abierta, más que un manual o pasos a seguir sin excepción.

6 A lo largo del texto se enuncian una serie de ejemplos, los cuales operan solo de modo ilustrativo. También se han creado para facilitar la comprensión de los constructos teóricos aquí presentados. 
relación con los hechos. Al iniciar las conversaciones trabajamos con el mapa de externalización, focalizando la reexperimentación intrusiva desde los contextos en los cuales se provocaban, conversando al principio de forma externalizada de la reexperimentación y, rápidamente, cambiando el foco hacia los pensamientos que permitían la emergencia de las imágenes intrusivas. Tal cambio de foco provino de una exploración detallada de las vivencias previas a los eventos de reexperimentación y permitió identificar patrones de pensamientos que eran temáticamente repetitivos, pero con contenidos disímiles.

Catalina definió que estos pensamientos se asimilaban a los personajes de la película Harry Potter - los dementors-, y se utilizó esta metáfora para nombrar cada una de las ideas asociadas a cada dementor, pudiendo identificar el dementor de la "culpa", el dementor de la "vergüenza", el dementor de la "propia incapacidad" y el dementor del "no servir para nada". Al indagar por la fuerza de cada dementor para invadir su vida, el dementor de la "propia incapacidad" asumió preponderancia por sobre los otros, lo que facilitó la comprensión de cómo emergía en particular. Catalina era invadida por reexperimentación intrusiva, al ser afectada por vivencias en las que ella no llenaba sus expectativas respecto de su desempeño en múltiples contextos sociales. Tales vivencias eran leídas desde esta idea de "no servir para nada", en la cual Catalina se sumergía en un remolino de aseveraciones respecto de su valía personal y una definición de sí misma como no merecedora de vivir. Este proceso era sumamente doloroso para ella y el único camino que vislumbraba como posible era el de hacerse daño, lo que le proveía una sensación momentánea de alivio. No obstante, la culpa que sentía posteriormente la invitaba a volver a un ciclo de dolor que se mantenía.

El trabajo en externalización permite, justamente, llegar a una posición de evaluar la influencia del problema en la vida de la persona, a la vez que tomar conciencia respecto de cómo procede el problema y de las estrategias que éste posee. En el caso de Catalina, "el no servir para nada" era una idea que propiciaba una mira- 
da monofocal, centrada en las sensaciones de ineficacia y en el no cumplimiento de sus propias expectativas, y especialmente en la de otros. Tal proceso rumiativo facilitaba una búsqueda activa de esta ineficacia, y Catalina exploraba las causas de esta definición en un esfuerzo por intentar explicarse y buscar solución a estas ideas. Lamentablemente para Catalina, tal proceso, por su aspecto centrado en lo negativo y sustentado en sus experiencias pasadas, la reconectaba con las experiencias de abuso, permitiendo que, inadvertidamente, se viese reexperimentando el abuso sexual como si estuviese sucediendo en el presente (Bustos y Paredes, 2018).

Creemos que la externalización es una práctica que permite a la persona evaluar su experiencia y significaciones asociadas. No obstante, en el contexto del trabajo en abuso sexual, existen conversaciones que pueden ayudar más a trabajar en la significación asociada a las recurrencias de patrones de pensamiento conectado a conclusiones de identidad deterioradas o focalizadas en lo negativo. Tal noción proviene de lo expresado con anterioridad, respecto del sentido que la mayoría de las personas proclives a rumiar tiende a atribuir al inicio de las elucubraciones. Esta intencionalidad no es abordada por otros modelos y creemos que es central para proveer de un contexto para cualquier trabajo terapéutico y/o psicoeducativo. White (2016) propone un mapa de conversación especialmente centrado en estados intencionales denominado "lo ausente pero implícito". Mediante esta noción se puede indagar sobre los significados propios de las personas, sobre sus sueños, valores y esperanzas, asociados a los actos cotidianos o cualquier acción que implique intencionalidad.

White, retomando las ideas de Bateson (1980) y Derrida (1978), genera la metáfora y el mapa de lo ausente pero implícito, con relación a cómo se da sentido a las cosas en el mundo, a la experiencia singular de las personas de realizar distinciones para explicarse el mundo y cómo esas distinciones dejan entrever un significado privilegiado o predominante, en contraposición a otros significados que quedan subyugados o que "quedan fuera" de las explicaciones. En esta diferencia emerge la posibilidad de lo no dicho, eso 
que está ausente, pero a la vez implícito. El mapa de "lo ausente pero implícito" (Carrey, Walther y Russel, 2010) propone un camino para iniciar una ruta conversacional, que ayuda a realizar una expresión de las emociones y sensaciones que se conectan con las acciones ligadas al problema. El propio acto de dar expresión a las luchas de la vida es un ejemplo de tomar posición, e implica un dar cuenta de las acciones que ya están siendo tomadas en relación con las descripciones preferidas de la vida y la identidad. Nombrar la respuesta y asociarla a habilidades o conocimientos que son expresados en la acción, permite abordar aquello a lo que se ha dado valor. Este acto de distinción significativo implica una historia social y relacional, que puede explorarse y que logra enriquecer aquellas definiciones preferidas del sí mismo de la persona, y que, finalmente, se pueden conectar a acciones a través del tiempo hacia el futuro.

Volviendo al ejemplo de Catalina, el trabajo descrito de externalización de la rumiación permitió generar una distancia entre lo pensado y su identidad, tomando una posición que la diferenciaba del pensamiento de no tener valía personal. No obstante, la ocurrencia continua de procesos rumiativos en los que el contenido era similar, nos motivó a explorar con Catalina la intencionalidad del inicio de dichos procesos. Al reconstruir un evento en el que se sentía rechazada en el plano escolar y que había culminado con el corte repetitivo de sus brazos en la misma noche, pudimos observar cómo iniciar los procesos rumiativos se relacionaba con la intención de batallar con la idea de que "no servía para nada". Al historiar esta lucha y su importancia en la vida, pudimos constatar las sensaciones de inmovilidad y frustración respecto del abuso de sus hermanos, y la sensación de culpabilidad y responsabilidad frente a estos hechos al sentir que no hizo lo suficiente para defenderlos. Tal idea se encontraba ligada, de una forma muy intrincada, con su compromiso de no permitir nunca más que algo les ocurriera, pero que, a la vez, la obligaba a una tiranía de "cumplir y servir para todo". No cumplir con estas expectativas en todo momento implicaba una confirmación de la idea de "no servir para 
nada". En el abordaje terapéutico se dio espacio a la construcción de identidad basada en el compromiso de ayudar y cuidar a sus hermanos, a su compromiso de protegerlos de futuros daños, pero también se trabajó respecto de esta tiranía de "cumplir y servir para todo" y como se relacionaba con las acciones de autoinjuria. Hubo conversaciones sobre alternativas para respetar la intención y el valor de "cuidar a sus hermanos", pero cuidando de que esta idea no se conectara con la tiranía de "cumplir y servir para todo". Se proyectó el futuro a partir de la noción enriquecida del "ayudar" y cómo esto se conectaba con sus proyecciones futuras de trabajar y estudiar una carrera relacionada con el prestar ayuda a los demás.

Las conversaciones respecto de "lo ausente pero implícito" logran dar un contexto a las acciones tomadas hasta el momento de dichas conversaciones, ligadas con el problema, pero dando luz a los estados intencionales que se contraponen al territorio del problema. No obstante, existen conversaciones que ayudan a rescatar alternativas con respecto a acciones, que nos pueden sumergir en mayores dificultades. Estas acciones son las que se han desarrollado a lo largo de la vida de la persona y que, a pesar de las tramas dominantes en relación con el problema, contradicen directamente las predicciones o imposiciones asociadas a las creencias del problema. Tales acciones se refieren a los desenlaces extraordinarios o excepciones, que hablan de mayor forma de conclusiones de vida distinta y que denotan habilidades únicas de las personas para lidiar con las dificultades que las atormentan. En el caso de Catalina, por ejemplo, implica conversar sobre momentos o eventos en los que Catalina no se dejó llevar por la tiranía de "cumplir y servir para todo", o momentos en que logró reconocer la sensación de vulnerabilidad asociada a percibir el inicio de la tiranía, pero que, en vez de cortarse, corrió donde su madre, la abrazó y le pidió que la cobijara. Tal acto, enriquecido por una descripción detallada de la toma de conciencia del problema y las estrategias de este, y el cómo ella respondió de una forma distinta, permitió hablar sobre habilidades únicas respecto de la conciencia de di- 
ficultad y qué la motivó a asistir a terapia, como también a pedir ayuda en vez de dar ayuda. Tal habilidad fue el inicio de conversaciones sobre lo que necesitaba para enfrentar la reexperimentación intrusiva e iniciar un camino conjunto de aprendizaje de técnicas relacionadas.

\section{Discusión}

Creemos que la terapia narrativa realiza un trabajo enriquecido en términos de construcción de sentido y permite una mayor alianza terapéutica que otros modelos en trauma, especialmente en ASI. $\mathrm{Al}$ dar énfasis, en una primera instancia, a conversaciones que pueden ser significativas y seguras para las personas, al no necesariamente centrarse en una narración de las experiencias traumáticas, sino más bien en poner énfasis en la agencia personal y en los estados intencionales de la persona. Sumado a ello, hay que mencionar y resaltar el hecho que la terapia narrativa es un abordaje que proviene desde el Trabajo Social, ya que tanto Michael White como David Epston son exponentes de la disciplina antes mencionada, lo cual posibilita un acercamiento a contextos terapéuticos tradicionalmente dominados por la psicología. En ese mismo sentido, ampliar las miradas y aproximaciones desde donde se "interviene" en trauma y abuso sexual, posibilita la inclusión de la disciplina del Trabajo Social, permitiendo democratizar los espacios de intervención/colaboración con personas que han experimentado situaciones límites y complejas. Cerrando esta idea, creemos que no solo la psicología y el Trabajo Social tienen algo que decir respecto de la intervención en trauma y el abuso, sino también la terapia ocupacional, la enfermería, la psiquiatría, entre otras. Mientras más y diversas disciplinas se preocupen del sufrimiento y el dolor humano, más posibilidades hay de integración y resignificación de la experiencia traumática; todos los aportes se pueden integrar en un quehacer informado y estratégicamente orientado.

Un elemento central en terapia narrativa es la noción de "agencia personal", entendida como la recuperación del control de la propia vida (White y Epston, 1993). Cuando se pierde o se reduce el apo- 
yo social, deberíamos intentar recuperar la agencia personal por la misma vía: la social y comunitaria. Por ello, la noción de "agencia personal" emerge posteriormente como un especial proceso de colaboración social, con la ayuda de los otros para recorrer un camino entre lo conocido y familiar, y lo posible de conocer respecto de sus vidas y su identidad preferida (White, 2016). La rumiación proviene de una intención de solucionar y enfrentar dificultades, de crear sentido a una vivencia intolerable. Abordar este sentido permite generar un contexto para acciones terapéuticas que faciliten la emergencia de nuevos conocimientos, ya sean conocimientos pasados de la persona o propuestos por algún otro dentro del entorno de la persona (incluidos los terapeutas).

Finalmente, la investigación que se asocie a aspectos de procesos entre rumiación, reexperimentación intrusiva y conductas problemáticas es primordial para mejorar los procesos terapéuticos de las personas que han sufrido abuso sexual infantil. Futuros estudios deberían presentar diseños longitudinales que permitan buscar asociaciones causales que pudiesen dar cuenta o no de las hipótesis esbozadas en este artículo. De la misma forma, investigación cualitativa tendiente a presentar hipótesis sobre los procesos y los discursos sobre éstos, es de suma importancia para teorizar respecto de la mejor forma de acompañar a las personas que han sobrevivido ASI.

\section{Bibliografía}

Aldao, A., Nolen-Hoeksema, S. \& Schweizer, S. (2010). Emotion-regulation strategies across psychopathology: A meta-analytic review. Clinical Psychology Review, 30, 217-237.

Alloy, LB., Abramson, LY., Hogan, M. E., Whitehouse, W. G., Rose, D. T., Robinson M. S. et al. (2000). The Temple-Wisconsin Cognitive Vulnerability to Depression Project: Lifetime history of Axis I psychopathology in individuals at high and low cognitive risk for depression. Journal of Abnormal Psychology, 109, 403-418.

Burwell, R. A. \& Shirk, S. R. (2007). Subtypes of Rumination in Adolescence: Associations Between Brooding, Reflection, De- 
pressive Symptoms, and Coping. Journal of Clinical Child and Adolescent Psychology, 36(1), 56-65.

Clark, D. M., Wells, A. (1995). A cognitive model of social phobia. In: R. G. Heimberg, M. R. Liebowitz, D. A. Hope \& F. R. Schneier (eds.), Social phobia: Diagnosis, assessment, and treatment. New York: Guilford Press New York.

Conway, M., Csabk, P., Holm, S. \& Blacke, C. (2000). On Assessing individual differences in rumination on sadness. J Pers Assess., 75(3): 404-425.

Coyle, E., Karatzias, T., Summers, A. \& Power, M. (2014). Emotions and emotion regulation in survivors of childhood sexual abuse: the importance of "disgust" in traumatic stress and psychopathology. European journal of psychotraumatology, 5. DOI: 10.3402/ejpt.v5.23306

Ehlers, A., Hackmann, A. \& Tanja, M. (2004). Intrusive Re-Experiencing in Post-Traumatic Stress disorder; Phenomenology, Theory, and Therapy. Memory, 12(4), 403-415.

Kross, E., Ayduk, O. \& Mischel, W. (2005). When asking "why" does not hurt. Psychological Science, 16, 709-715.

Lyubomirsky, S., Caldwell, N. D. \& Nolen-Hoeksema, S. (1998). Effects of ruminative and distracting responses to depressed mood on retrieval of autobiographical memories. Journal of Personality and Social Psychology, 75, 166-177.

Lyubomirsky, S., Tucker, K. L., Caldwell, N. D. \& Berg, K. (1999). Why ruminators are poor problem solvers: Clues from the phenomenology of dysphoric rumination. Journal of Personality and Social Psychology, 77, 1041-1060.

McLaughlin, K. A., Borkovec, T. D. \& Sibrava, N. J. (2007). The effects of worry and rumination on affect states and cognitive activity. Behavior Therapy, 38, 23-38.

Nolen-Hoeksema, S., Blair E., Wisco, B. \& Lyubomirsky, S. (2008). Rethinking Rumination. Perspective of Psychological Science, (3-5), 400-424.

Sarin, S. \& Nolen-Hoeksema, S. (2010). The dangers of dwelling: An examination of the relationship between rumination and consumptive coping in survivors of childhood sexual abuse. Cognition and Emotion, 24(1), 71-85.

Smith, J. M. \& Alloy, L. B. (2009). A roadmap to rumination: A review of the definition, assessment, and conceptualization of this multifaceted construct. Clin Psychol Rev., 29(2), 116-128.

Veale, D. (2008). Behavioural activation for depression. Advances in Psychiatric Treatment, 14, 29-36. 
Watkins, E. \& Moulds, M. (2005). Distinct modes of ruminative selffocus: Impact of abstract versus concrete rumination on problem solving in depression. Emotion, 5, 319-328.

White, M. (2002) Narratives of therapist lives. Barcelona, España: Gedisa.

White, M. (2004). Guías para una Terapia Familiar Sistémica. Barcelona: Gedisa.

White, M. (2016). Mapas de la práctica Narrativa. Santiago, Chile: Pranas.

White, M. \& Epston, D. (1993). Medios Narrativos para fines Terapéuticos. Barcelona, España: Gedisa.

Zhan, J., Tang, F., He, M., Fan, J., Xiao, J., Liu, C. \& Luo, J. (2017). Regulating Rumination by Anger: Evidence for the Mutual Promotion and Counteraction (MPMC) Theory of Emotionality. Front Psychol., 8, 1871.

DOI: 10.3389/fpsyg.2017.01871 